\title{
Rânula bilateral com recidiva em glândula mandibular e sublingual em cão*
}

\section{Ranula bilateral with recurrence in submandibular gland and sublingual in dog}

\author{
Vanessa Chrystina Pontes da Silva Gomes, ${ }^{* *}$ Giovanna Carla de Oliveira Campos, ${ }^{* *}$ Pedro Gustavo Santos Nóbrega, ${ }^{* *}$ \\ Kalyne Danielly Silva de Oliveira, ${ }^{* *}$ Lennon Magno Alves do Nascimento, ${ }^{* *}$ \\ Thamires Barreto Silva Souza, ${ }^{* *}$ Eraldo Barbosa Calado**
}

\begin{abstract}
Resumo
A mucocele sublingual é uma afeç̧ão incomum em cães, porém de grande importância para a medicina veterinária, devido aos prejuízos gerados por suas consequências. Deste modo, o presente relato tem como objetivo descrever o caso de mucocele sublingual em cão com posterior recidiva em glândula mandibular e sublingual, bem como discutir sua importância para a medicina veterinária. Foi atendido no Hospital Veterinário Dix-Huit Rosado Maia - HOVET/ Universidade Federal Rural de Semiárido (UFERSA), um cão macho, com um ano e 10 meses de idade, apresentando aumento de volume bilateral na região sublingual há, aproximadamente, dois meses. Após exame clínico suspeitou-se de mucocele da glândula sublingual bilateral (rânula) indicando-se o tratamento de ressecção da glândula sublingual esquerda devido ao aspecto nodular e marsupalização da glândula sublingual direita. Passado um mês da alta médica o animal retornou ao HOVET - UFERSA com queixa de aparecimento de um novo aumento de volume sublingual e na região cervico-ventral suspeitando-se de uma possível mucocele cervical e recidiva da mucocele sublingual esquerda. Indicou-se como tratamento a sialoadenectomia sublingual e mandibular. Cerca de dez dias após a última cirurgia o cão não apresentava mais aumento de volume da área afetada e não havia sinais de alteração quanto à produção de saliva. $\mathrm{O}$ tratamento sugerido proporcionou bons resultados promovendo a reparação tecidual e reestruturação do local afetado e diminuindo o índice de recidiva.
\end{abstract}

Palavras-chave: glândula salivar, marsupialização, mucocele.

\begin{abstract}
The sublingual mucocele is a rare disease in dogs, but very important for veterinary medicine due to losses generated by its consequences. Thus, this report aims to describe the case of sublingual mucocele in a dog with subsequent relapse in mandibular and sublingual gland and discuss their importance to veterinary medicine. It was the Veterinary Hospital Dix-Huit Rosado Maia HOVET / Universidade Federal Rural de Semi-árido (UFERSA), a male dog with one year and 10 months, an increase of bilateral volume in the sublingual region for approximately two months. After clinical examination was suspected of mucocele of bilateral sublingual gland (ranula) indicating the treatment of sublingual left gland resection due to nodular and marsupalização the right sublingual gland. After one month of a medical release the animal returned to HOVET - UFERSA with emergence of complaint from a further increase in sublingual volume and cervical-ventral region suspecting it is a possible cervical mucoceles and recurrence of sublingual mucocele left. It is stated as a treatment to sublingual and mandibular sialoadenectomy. About ten days after the last surgery, the dog had no more swelling of the affected area and no signs of change with saliva production. However, the suggested treatment provided good results by promoting tissue repair and restructuring of the affected area and decreasing the recurrence rate.
\end{abstract}

Keywords: Salivary gland, Marsupialization, Mucocele

\section{Introdução}

A mucocele sublingual é uma afeç̧ão pouco frequente em cães, mas de grande importância para a medicina veterinária, pois, apesar de não ser considerada uma emergência clínica-cirúrgica, as consequências geradas pelo edema e compressão regional se tornam um problema, além da dor decorrente da resposta inflamatória (Gioso, 2007). Este termo consiste em uma retenção de saliva localizada em uma região anormal, resultante do extravasamento da glândula salivar ou de seu ducto em virtude de algum tipo de injúria (Kazemi et al., 2012). São caracterizadas clinicamente por aumento de volume progressivo e regular, uni ou bilateral, de tamanhos distintos, não aderido, circunscrito, não invasivo, de superfície lisa, indolor à palpação, hipertérmicos ou não, flutuante e flácidos (Vallefuoco et al., 2011) e revestido por epitélio pseudoestratificado (Gioso, 2007). Podem ser

*Recebido em 10 de fevereiro de 2017 e aceito em 9 de maio de 2017.

**Universidade Federal Rural do Semiárido UFERSA.

Autor para correspondência: nessinhachrystina@gmail.com 
formadas na região cervical ventral, sublingual, faríngea, parotídea ou zigomática, de acordo com a glândula e ducto salivar afetado (Fossum, 2002; Kazemi et al., 2012). Em um mesmo animal pode ocorrer mais de um tipo de mucocele concomitantemente, sendo denominadas de mucoceles complexas. E quando localizadas na região sublingual, são denominadas de rânulas (Andrade et al., 2011; Fossum, 2002).

Seu diagnóstico é fácil e definitivo. Entretanto, o clínico deve estar atento para sua ocorrência de forma a diagnosticá-la precocemente para que o tratamento cirúrgico seja efetuado com sucesso. Dentre os tratamentos mais recomendados para tal afecção relata-se a drenagem de seu conteúdo com a, marsupialização da glândula (Pignone et al., 2009; Gioso, 2007) e/ ou a exérese da glândula salivar acometida (Vale et al., 2009, Wiggs e Lobprise, 1997), além de obliteração do ducto salivar correspondente (Andrade et al., 2011)

Objetivou-se, descrever caso de mucocele sublingual em cão com recidiva em glândula mandibular e sublingual, atendido no Hospital Veterinário Dr. Jerônimo Dix-Huit Rosado Maia da Universidade Federal Rural do Semiárido - UFERSA.

\section{Relato de caso}

Foi atendido no Hospital Veterinário Dix-Huit Rosado Maia - HOVET/ UFERSA, um cão macho, sem raça definida, com 1 ano e 10 meses de idade, apresentando salivação excessiva, dificuldade para se alimentar e aumento de volume bilateral na região sublingual há aproximadamente dois meses. Através do exame clínico constatou-se uma massa ulcerada de consistência mais firme localizada na face ventral esquerda da língua (Figura 1A) e um aumento de volume apresentando conteúdo líquido na face ventral direita (Figura 1B). Para esclarecimento solicitou-se exame de citologia aspirativa por agulha fina (CAAF) que evidenciou presença de conteúdo salivar. A citologia da secreção salivar revelou a presença de células epiteliais glandulares, além de macrófagos e neutrófilos, concluindo-se ser uma mucocele da glândula sublingual.

Baseando-se na anamnese, no exame físico e complementar, definiu-se o diagnóstico de mucocele da glândula sublingual bilateral (rânula) indicandose o tratamento de exérese da glândula sublingual esquerda devido ao aspecto nodular e marsupalização da glândula sublingual direita. Como medida préoperatória, preconizou-se os exames de hemograma, função renal, função hepática e avaliação cardiológica por eletrocardiograma e radiografia, os quais evidenciaram alteração de enzimas hepáticas. Instituiu-se o início do tratamento com SAME (20 $\mathrm{mg} \cdot \mathrm{kg}^{-1}$ ) associado a Silimarina $\left(20 \mathrm{mg} \cdot \mathrm{kg}^{-1}\right)$ e ao exame cardiológico observaram-se alterações como cardiomegalia e leve bradicardia. Após estabilização das alterações fisiológicas o paciente foi encaminhando para a intervenção cirúrgica.

Na medicação pré-anestésica, foi administrado acepromazina $\left(0,07 \mathrm{mg} \cdot \mathrm{kg}^{-1}\right)$ associada com morfina $\left(0,4 \mathrm{mg} \cdot \mathrm{kg}^{-1}\right)$, por via intramuscular. Em seguida o animal foi preparado para cirurgia e levado para o centro cirúrgico, onde foi induzido com propofol (4 mg. $\mathrm{kg}^{-1}$, por via intravenosa) e a manutenção anestésica feita com isoflurano diluído em oxigênio a $100 \%$, em sistema semifechado. $\mathrm{O}$ animal foi posicionado em decúbito dorsal e através da tração no sentido rostral da língua, observamos a presença de rânulas bilaterais, o acesso cirúrgico para exérese da glândula sublingual esquerda foi realizado segundo a técnica preconizada por Fossum (2002), por meio de uma incisão elíptica ao redor da rânula, em seguida procedeu à exposição da glândula sublingual e exérese da mesma, com obliteraçãodo ducto salivar correspondente (Andrade et al., 2011; Gioso, 2003), seguido de rafia da mucosa com fio de ácido poliglicolico (vicryl) 3-0 em padrão simples separado (Figura 2).
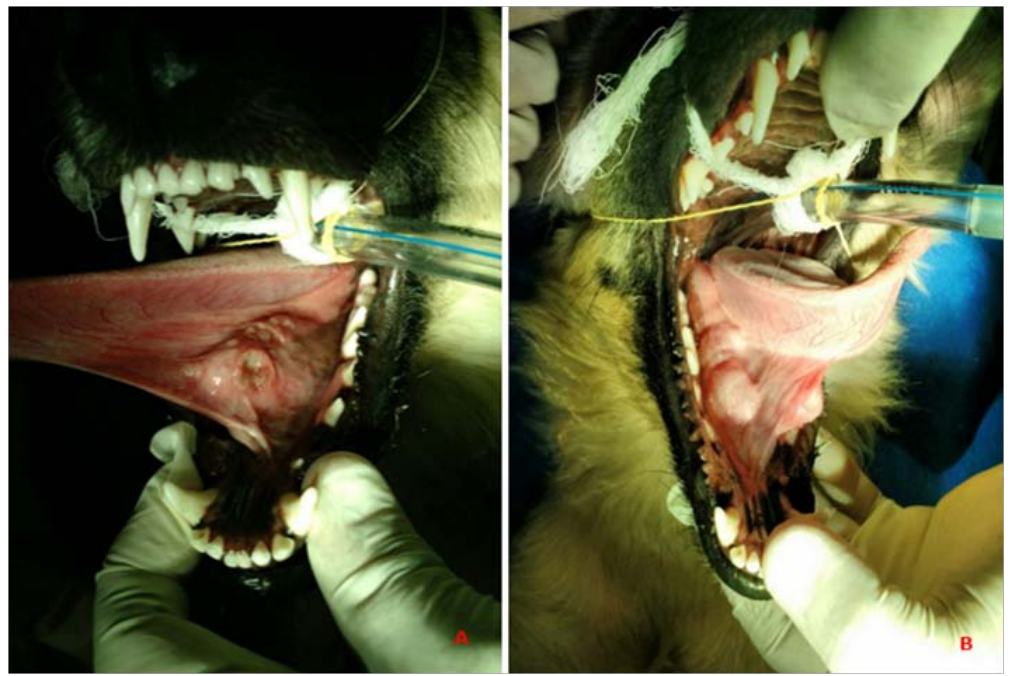

Figura 1: A) Massa ulcerada de consistência mais firme localizada na face ventral esquerda da língua. B) Aumento de volume macio apresentando conteúdo líquido na face ventral direita.

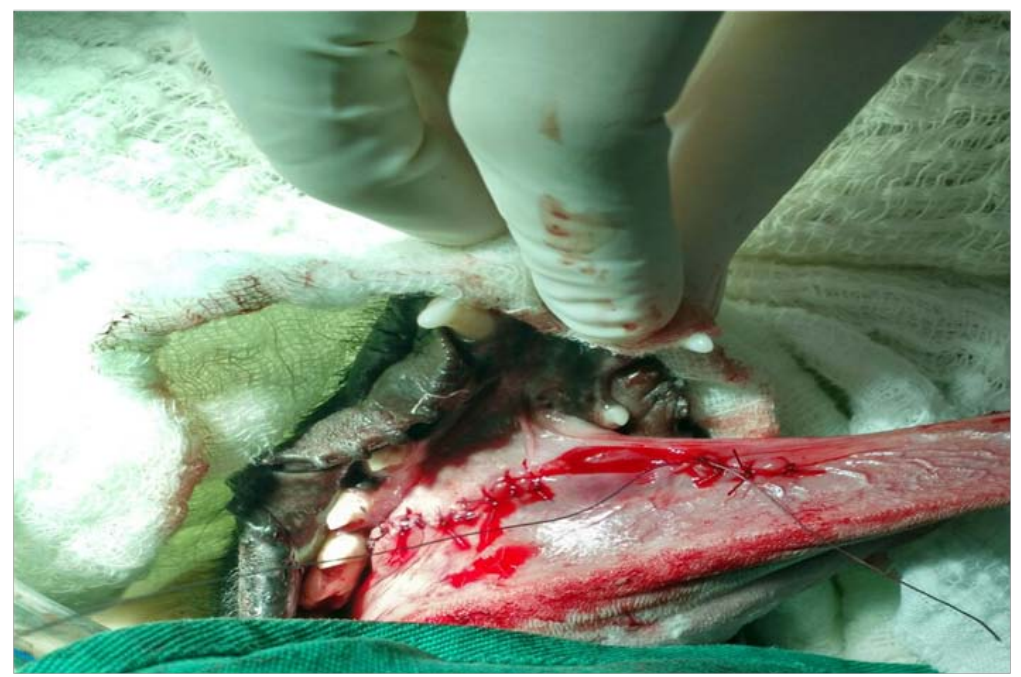

Figura 2: Rafia da mucosa após exérese da glândula sublingual

A glândula sublingual direita foi marsupializada através da técnica preconizada por Fossum(2002), com uma incisão elíptica na mucosa procedeu a drenagem do conteúdo salivar. 
Após drenagem completa foi realizada a sutura da mucosa ao revestimento da mucocele com fio de sutura 3-0 vicryl em padrão simples separado (Figura 3). Todo o material da exérese foi imerso em formaldeído $10 \%$ e encaminhado para exame histopatológico.

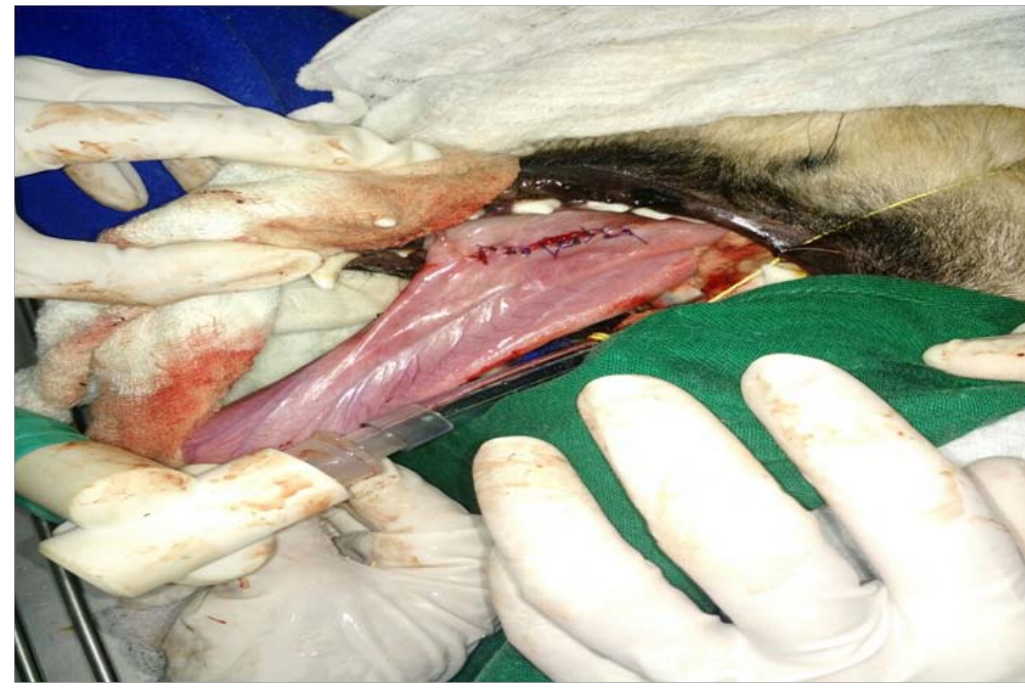

Figura 3: Marsupialização da glândula sublingual direita.

Para o pós-operatório foi prescrito cefalexina $\left(25 \mathrm{mg} \cdot \mathrm{kg}^{-1}\right.$, por via oral) a cada 12 horas durante dez dias, metronidazol (10 mg. $\mathrm{kg}^{-1}$, por via oral) a cada 12 horas durante sete dias, dipirona sódica (25 mg. kg-1 , por via oral) a cada 8 horas durante quatro dias, Oncilon-A (no local da ferida cirúrgica) e dieta pastosa durante cinco dias. Depois de 10 dias da intervenção cirúrgica, o animal foi reavaliado e constatou-se que estava em bom estado, sendo liberado para exercer suas atividades normalmente.

Após um mês da alta médica, retornou ao HOVET - UFERSA com queixa de aparecimento de um novo aumento de volume sublingual e da região cervico-ventral. Através da palpação constatou-se que a massa apresentava-se com conteúdo líquido o qual foi realizado uma punção aspirativa constatando a presença de saliva na região. Constatandose recidiva da mucocele, ficando indicado como tratamento a sialoadenectomia das glândulas sublingual e mandibular para evitar recidivas.

Como medida pré-operatória preconizou-se a realização de exames pré-cirúrgicos básicos necessários (hemograma, função renal, função hepática), os quais não evidenciaram nenhuma alteração e o animal foi encaminhado para cirurgia. $O$ paciente foi submetido à anestesia geral inalatória e posicionado em decúbito lateral esquerdo. $\mathrm{O}$ acesso cirúrgico foi realizado segundo a técnica preconizada por Pignone (2009) através de uma incisão de pele na região do aumento de volume ventral ao pescoço divulsionando e identificando a glândula acometida. Após identificar a glândula mandibular entre os ramos das veias linguofacial e maxilar, isolou-se o seu ducto, procedendo-se a dissecção em direção à porção monostomática da glândula sublingual. Em seguida, o ducto foi ligado o mais medial possível e o complexo de glândulas removido. (Figura 4).
A sutura foi realizada em três planos, de acordo com Fossum (2002): um plano interno com fio absorvível 3-0 (vicryl) aproximando o tecido mais profundo a cápsula em pontos simples minimizando o "espaço anatômico". O segundo plano foi realizado com fio absorvível 3-0 (vicryl) aproximando os músculos superficiais e tecido subcutâneo. E o terceiro plano foi realizado com fio de náilon 3-0 em padrão simples separados para fechamento da pele. Antes de se iniciar a aproximação do tecido subcutâneo, realizou-se a colocação e fixação de um dreno. Após o término realizou-se bandagem com leve compressão na região.

Foi prescrito de pós-operatório dipirona sódica (25 mg. $\left.\mathrm{kg}^{-1}\right)$ e cloridrato de tramadol $\left(2 \mathrm{mg} \cdot \mathrm{kg}^{-1}\right)$ a cada oito horas por quatro dias; meloxicam $(0,1$ $\mathrm{mg} \cdot \mathrm{kg}^{-1}$ ) uma vez ao dia por três dias, amoxicilina + clavulanato de potássio $\left(20 \mathrm{mg} \cdot \mathrm{kg}^{-1}\right)$ a cada 12 horas por dez dias., todos por via oral. Após cinco dias da cirurgia, o animal apresentou edema na região rostral e pelo dreno apresentou secreção serossanguinolenta. Removeu-se o dreno e recomendou-se ao proprietário continuar com o meloxicam por mais três dias e limpeza tópica com solução fisiológica e clorexidine aquosa $2 \%$ (merthiolate ${ }^{\circledR}$ ) na área de inserção do dreno. Depois de 15 dias da intervenção cirúrgica realizou-se a retirada dos pontos e o animal foi reavaliado constatando-se que estava em bom estado, recebendo alta médica.

\section{Resultados e discussão}

Afecções que envolvem as glândulas salivares em cães são incomuns e apresentam uma baixa incidência, de aproximadamente 0,3\% (ANDRADE et al., 2011). De acordo com Fossum (2007) e Manfra-Marretta (2006), o aumento de volume é comumente observado na região cervical, sublingual e faríngea. No presente caso, ocorreu inicialmente sublingual, recidivando em região cervical e sublingual.

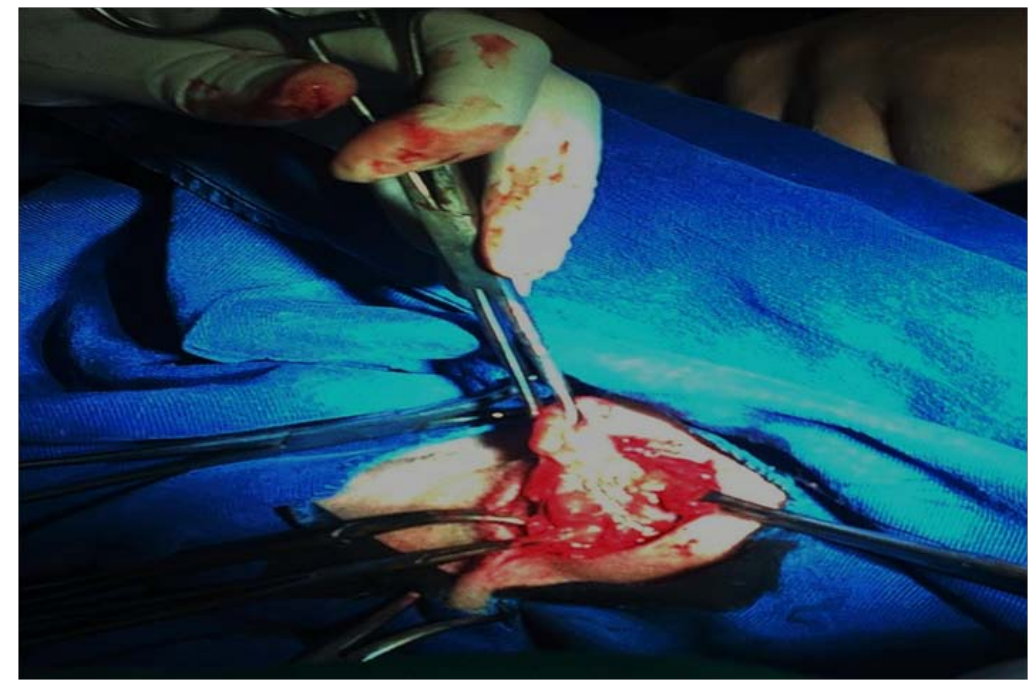

Figura 4: Identificação a glândula acometida. 
Para o diagnóstico da mucocele salivar cervical, na palpação da região em decúbito dorsal pode indicar qual lado há acúmulo salivar (Gioso, 2007). Concorda-se que o aumento de volume na região ventral do pescoço pode caracterizar diversas etiologias a exemplo mucocele, neoplasia, abscessos e hematomas (Hedlund e Fossum, 2007), ficando indicado a realização de punção para análise histológica, conforme realizado no paciente. Em casos de mucocele o conteúdo pode apresentar coloração de amarelo-palha a avermelhada, de consistência viscosa e microscopicamente observa-se presença de saliva e poucas células sanguíneas, assim como foi encontrado no caso relatado (Gioso, 2007; Hedlund e Fossum, 2007).

Apesar de a marsupialização estar dentro das técnicas de tratamento realizadas, a exérese cirúrgica das glândulas é o tratamento definitivo recomendado quando há recidiva, pois além de promover a reestruturação da área afetada também impede que elas ocorram (Andrade et al., 2011).

Sendo assim, pôde-se verificar que o tratamento da mucocele conforme descrito anteriormente apresenta resultados favoráveis com a excisão das glândulas salivares envolvidas, haja vista que o tratamento conservador de punção aspirativa do conteúdo salivar anteriormente ao tratamento cirúrgico não resolve o processo (Gioso, 2007).

Preconiza-se que, ao se realizar uma sialoadenectomia, preserve a cápsula da glândula, evitando extravasamento de saliva, o qual não se conseguiu realizar neste caso (Hedlund e Fossum, 2007; Wiggs e Lobprise, 1997). Segundo Gioso (2007) o dreno de penrose é recomendado nos casos de sialoadenectomia

\section{Referências}

ANDRADE, E. C.; SEPÚLVEDA, R. V.; GALVÃO, S. R.; DEL CARLO, R. J. Ressecção bilateral de glândulas salivares no tratamento da sialocele cervical em cão. Relato de caso. Revista do CFMV - Conselho Federal de Medicina Veterinária, v. 17, n. 54, p. 44-48, 2011.

DIAS, F. G. G.; PEREIRA, L. F.; SANTILLI, J.; MAGALHÃES, G. M.; GONÇALVE, DIAS, L. G. G. G. Mucocele em cães. Enciclopédia Biosfera, Centro Científico Conhecer - Goiânia, v. 9, n. 16, p.1534,2013.

FOSSUM, T. W. Cirurgia de pequenos animais. In: Cirurgia do Sistema Digestório. São Paulo: Roca, Cap. 16, p. 222-405. 2002.

FREITAS, S. E. N.; FURUSE, C. F.; BIAZOLLA, E. R.; ANDRADE SOBRINHO, J. Rânula: técnica de marsupialização. Relato de caso clínico. Revista Odontológica de Araçatuba, v. 25, n.1, p. 53-56. 2004.

GIOSO, M.A. Glândulas Salivares. In: Odontologia para o clínico de pequenos Animais. 2. ed. São Paulo: Manole, p. 109-112, 2007.

KAZEMI, D.; DOUSTAR, Y.; ASSADNASSAB, G. Surgical

treatment of a chronically recurring case of cervical mucocele in a German shepherd dog. Case Reports in Veterinary Medicine, v. 1, n.1, p. 1-4, 2012. das glândulas mandibular e sublingual, prevenindo a formação de seroma, podendo permanecer de 24 a 72 horas. No caso relatado, foi colocado um dreno no paciente utilizando-se tubo de PVC $12 \mathrm{~mm}$, o qual permaneceu durante 72 horas, sendo removido em seguida.

Analisando a citologia do nódulo sublingual revelou presença de células mesenquimais, anisocitose e anisocariose moderadas. Neutrófilos, linfócitos e debris celulares de permeio, levando a diagnóstico sugestivo de neoplasia mesenquimal maligna, que foi descartada após exame histológico da amostra coletada na cirurgia.

Os resultados histológicos do nódulo sublingual indicaram reação inflamatória crônica ativa proliferativa por ruptura de ducto salivar compatível com rânula/ sialocele inflamada, não evidenciando sinais de transformação ou infiltração neoplásica. Cerca de dez dias após cirurgia de sialoadenectomia o cão não apresentava mais aumento de volume da área afetada e não havia sinais de alteração quanto à produção de saliva.

\section{Conclusão}

O diagnóstico precoce e a terapia cirúrgica correta nos casos de mucocele de qualquer natureza são fatores determinantes para o prognóstico e aumento da qualidade de vida dos cães acometidos. No entanto, recidivas podem ocorrer caso a glândula salivar não seja totalmente removida. Neste caso, a exérese da glândula salivar afetada seja a conduta mais eficiente, por promover a reparação tecidual, reestruturação do local afetado e diminuição da possibilidade de recidiva.

MANFRA-MARRETTA, S. Dentistry and diseases of the oropharynx, In: Saunders manual of Small Animal Practice, Saunders, 3. ed, St. Louis, Mo, USA, p. 609-635. 2006.

PIGNONE, V. N.; FARACO, C. S.; ALBUQUERQUE, P. B.; RECLA, G.; GIANOTTI, G.; CONTESINI, E. A. Sialólito no ducto da glândula mandibular em cão. Acta Scientiae Veterinariae. v. 37, n. 3, p. 273-280, 2009.

VALE, D. F.; RAMOS, R. M.; FERREIRA, F. S.; QUEIROZ, F. F.; SAMPAIO, R. L.; LACERDA, M. S. S. Sialolitíase em ducto da glândula submandibular em cão. Jornal Brasileiro de Ciência Animal, v. 2, n. 3, p. 1-2, 2009.

VALLEFUOCO, R.; JARDEL, N.; EL MRINI, M.; STAMBOULI, F.; CORDONNIER, N. Parotid salivary duct sialocele associated with glandular duct stenosis in a cat - case report. Journal of Feline Medicine and Surgery, v. 13, n. 1, p. 781-783. 2011.

WIGGS R.; LOBPRISE H. Veterinry Dentistry - Principles e Practice. Philadelphia: Lippincott - Raven. p. 748. 1997. 